\title{
Faire exister un intellectuel collectif dans le champ politique
}

Prises de position et mobilisation partisane au sein du Rassemblement démocratique révolutionnaire (France, fin des années 1940)

Creating a Collective Intellectual in the Political Field. Positionings and partisan activism within the Rassemblement Démocratique Révolutionnaire (France, late 1940's)

\section{Bastien Amiel}

\section{OpenEdition}

Journals

\section{Édition électronique}

URL : https://journals.openedition.org/bssg/673

DOI : $10.4000 /$ bssg.673

ISSN : 2490-9424

\section{Éditeur}

Presses universitaires de Vincennes

Référence électronique

Bastien Amiel, «Faire exister un intellectuel collectif dans le champ politique », Biens Symboliques / Symbolic Goods [En ligne], 8 | 2021, mis en ligne le 20 mai 2021, consulté le 23 juillet 2021. URL : http:// journals.openedition.org/bssg/673; DOI : https://doi.org/10.4000/bssg.673

Ce document a été généré automatiquement le 23 juillet 2021. 


\section{Faire exister un intellectuel collectif dans le champ politique}

Prises de position et mobilisation partisane au sein du Rassemblement démocratique révolutionnaire (France, fin des années 1940)

Creating a Collective Intellectual in the Political Field. Positionings and partisan activism within the Rassemblement Démocratique Révolutionnaire (France, late 1940's)

\section{Bastien Amiel}

\section{NOTE DE L'AUTEUR}

L'auteur remercie Mathilde Sempé, Paul Lehner et Jean-Baptiste Paranthoën pour leurs relectures successives ainsi que les experte's mandatée's par la revue pour leurs commentaires et leurs suggestions précieuses.

1 Le Rassemblement démocratique révolutionnaire (RDR), entreprise politique créée en France en février 1948 semble concentrer tous les stigmates de l'échec politique. Son recrutement n'a pas dépassé les 2000 membres, son activité n'a pas duré plus de deux ans et sa postérité, tant intellectuelle que partisane, est minime (Birchall 1999). Pour autant, nous formons l'hypothèse que cette entreprise politique, par son recrutement principalement intellectuel, le répertoire d'action mobilisé par ses membres, mais également ses prises de position politique et sa production idéelle, permet d'enrichir l'analyse des relations qu'entretiennent les intellectuelle's à l'engagement politique ainsi qu'à la recomposition des champs intellectuel et politique au début de la guerre froide en France. L'étude des logiques de recrutement, de mobilisation et de production d'un discours politique original du RDR relève d'une perspective de sociologie historique du politique et renvoie à deux approches ici complémentaires.

2 Nous nous inscrivons dans la lignée du « tournant sociologique » qu'a connu l'étude des partis politiques durant la décennie 1980. Il consiste dans le remplacement de l'étude 
des partis comme mise en pratique d'une conviction, par celle de leur dimension entrepreneuriale (Offerlé 1987), en prenant en compte la sociologie de leurs membres (Pudal 1989) et en les inscrivant dans un environnement local, social et politique précis (Sawicki 1997). Une telle perspective revient finalement à mettre à distance les implicites qui fondent une explication des pratiques politiques par la rationalité supposée des acteurs sociaux (Gaxie 2003).

3 Compte tenu de la spécificité du recrutement social des membres du RDR, que nous aurons l'occasion d'expliciter plus loin, ce travail relève également d'une sociologie historique de l'engagement des intellectuelle's. En effet, depuis les travaux portant sur l'affaire Dreyfus et l'invention, par la confrontation au politique, de la figure de l'intellectuel (Charle 1990), de nombreuses études ont investi ce domaine de recherche. En prenant pour objet des groupes d'intellectuelle-s confrontée's à des évènements, depuis l'étude des intellectuelle's antifascistes de l'entre-deux-guerres (Racine \& Trebitsch 1994) jusqu'aux écrivain'e-s en mai-juin 1968 (Gobille 2018) en passant par celles et ceux qui agissent durant la période de l'Occupation (Sapiro 1999), ces études ont actualisé les enjeux sociologiques de l'analyse des engagements intellectuels en fonction de contextes spécifiques. D'autres études ont privilégié l'observation et l'analyse de certains collectifs intellectuels comme les surréalistes (Bandier 1999; Reynaud-Paligot 2001) ou les situationnistes (Brun 2014) dans la construction d'un rapport au politique, ou encore l'étude d'une idéologie politique comme le communisme du point de vue de la gestion de ses partisane's intellectuelle-s (Matonti 2005 ; Gouarné 2013). Enfin, les travaux d'Éric Agrikoliansky sur la Ligue des droits de l'homme ou de Philippe Gottraux sur le groupe « Socialisme ou Barbarie » ont pris le parti d'investiguer des organisations politiques investies par des intellectuelle's (Agrikolianski 1997 ; Gottraux 1997).

Ces travaux ont contribué à forger collectivement une grille d'analyse heuristique pour l'étude des formes de transgression des frontières symboliques entre les champs politique et intellectuel. En considérant les logiques de mobilisation des intellectuelle's non plus seulement en fonction des convictions - ce qui doublerait la croyance dans leur rationalité d'une autre impasse qu'est celle de l'intellectuelle conçu'e comme entité surplombante (Bourdieu 1977) - mais comme le résultat de positions acquises dans leur champ d'origine, ces travaux ont en effet apporté une contribution stimulante tant à l'étude de l'engagement intellectuel qu'à celle des mobilisations partisanes.

5 Le RDR illustre à son tour l'intérêt de ces approches. Nous l'aborderons en effet comme l'espace d'investissement partisan d'intellectuelle's cherchant à construire une entreprise politique susceptible de rendre possible une double action, intellectuelle et politique, sans que l'une ou l'autre de ces logiques ne prenne le pas sur l'autre. À ce titre, la participation de Jean-Paul Sartre, intellectuel dominant de l'époque, est significative puisqu'elle ne se limite pas à un soutien symbolique mais prend la forme d'un engagement militant, financier et pratique (Amiel 2013). C'est en observant les fluctuations historiques entre champ politique et champ intellectuel que l'on peut comprendre qu'après l'aboutissement des processus longs de différenciation (Dulong 2010) et de professionnalisation (Offerlé 1999) du champ politique en France, la période de la Libération apparaît comme une conjoncture de désectorisation des logiques sociales (Dobry 1992), qui rend dès lors pensable un tel équilibre pour les membres du RDR (Amiel, Sempé, Dirkx 2015). Il faut par ailleurs avoir à l'esprit l'histoire longue de 
la structuration du champ intellectuel en France et en Europe (Charle \& Jeanpierre 2016) et les effets que la massification scolaire, entre autres facteurs, entraîne sur une conception de plus en plus collective de la pratique intellectuelle (Joly 2018) dont il est possible de concevoir le RDR comme un indice supplémentaire.

6 Dans cet article nous essaierons en outre de comprendre dans quelle mesure la tentative de créer une entreprise partisane par des intellectuelle-s peut peser sur le processus de mise en politique des idées (Skornicki \& Tournadre 2015 : 93). Notre travail fait écho en effet à de récentes études intéressées à rendre raison des mécanismes d'appropriation partisane des idées politiques (Rioufreyt 2019), ou qui s'attachent à la circulation des idées en milieu partisan concernant les enjeux économiques dans le Parti communiste français (Azam 2019) et la Section française de l'internationale ouvrière (SFIO) (Fulla 2019), les conditions de félicité de la notion de " grand remplacement» au sein du parti Alternativ für Deutschland (Alternative pour l'Allemagne) (Leconte 2019), les questions de "sécurité » au sein du Parti socialiste en France (Cos 2019) ou encore la (re)définition du conservatisme au sein du parti Russie unie (Fauconnier 2019). Ces travaux participent à une certaine reformulation de l'approche idéelle en milieu partisan dans le contexte d'un mouvement national (Pudal 2006; Matonti 2012; Skornicki \& Gaboriaux 2017) et international (Skinner 2001) de renouveau des études historiques et sociologiques sur les idées politiques comme discours historiquement et socialement situés.

7 C'est donc en croisant l'analyse des pratiques politiques de ses membres et celle de sa production de discours que nous nous intéresserons, dans la perspective d'une sociologie historique des idées politiques qui se donne pour ambition de penser la production de bien symboliques en fonction de contraintes sociales (Belorgey, Chateigner, Hauchecorne, Pénissat 2011), à la spécificité de la production idéologique et des formes de mobilisation du RDR. La particularité du recrutement social des membres de celui-ci, et plus encore de ses dirigeants, pour la très grande majorité des intellectuelless, suggère la production d'idées politiques par des membres dont les pratiques professionnelles non partisanes relèvent également de la production, de la diffusion et de la manipulation de biens symboliques. Nous verrons cependant qu'une telle surreprésentation n'est pas seulement à l'origine de la production et de la diffusion de biens symboliques, mais aussi d'une constante indétermination dans la définition du RDR par ses membres - entre parti politique et rassemblement d'intellectuelles.s. Le Rassemblement apparait dès lors comme le résultat d'une dynamique de mobilisation instable qui s'explique pour partie par un répertoire de l'action collective oscillant entre les logiques traditionnelles de l'engagement intellectuel, associées à la légitimité nominative de ses membres, et celles de l'engagement partisan renvoyant au nombre de ses adhérente's (Gobille $2008: 62$ ).

8 Les prises de position politique des membres du RDR et ses formes de mobilisation seront abordées à partir de l'analyse de différentes sources archivistiques du Rassemblement. Les « archives du RDR » n'existent pas en propre, ce qui constitue un indice de son faible degré d'institutionnalisation (Perrot 1993: 18), mais on trouve cependant, éclaté dans plusieurs lieux de conservation, notamment militants, du matériel partisan susceptible de donner accès aux idées produites par le groupe. C'est par la combinaison de deux types de sources, interne et externe, que nous aborderons les idées du RDR. Le premier renseigne les débats internes et la construction concurrentielle de l'idéologie du groupe grâce à deux publications, Les cahiers $d u$ 
propagandiste (un numéro en septembre 1948) et le Bulletin intérieur (trois numéros en janvier, février et mai 1949) ${ }^{1}$. Le second donne accès aux différentes formes de discours politique tenus publiquement par le groupe ou en son nom. Il regroupe la collection du bimensuel du Rassemblement, La Gauche, dont les quatorze numéros, disponibles à La Contemporaine ${ }^{2}$, ont été publiés entre mai 1948 et mars 1949, et donnent notamment accès à l'ébauche de « programme » du RDR; ainsi que « l'appel pour un rassemblement démocratique révolutionnaire » ayant initié l'entreprise politique le 28 février 1948 ; ou encore la profession de foi produite par Charles Marché, candidat du RDR aux élections cantonales des 20 et 27 mars $1949^{3}$. Enfin, l'ouvrage Entretiens sur la politique, supposé livrer les prises de position politique de trois des dirigeants du RDR, parmi les plus célèbres, sera également étudié. Le contexte au sein duquel ces discours sont produits est par ailleurs restitué par l'exploitation d'autres archives, comme celles des renseignements généraux de la Seine ou de publications médiatiques contemporaines comme le journal Franc-Tireur auquel une partie des membres du RDR appartient. Après avoir décrit la composition sociale du RDR et les enjeux politiques et intellectuels qui découlent de cette tentative de faire exister une position neutraliste nouvelle au sein du champ politique français (Milza 1987), la conjoncture de guerre froide nous amènera à interroger les prises de position politique des membres du RDR et les formes particulières d'une mobilisation duale : d'un côté les intellectuelle's qui reproduisent, souvent en dehors du collectif, des pratiques de production de bien symboliques routinières (Ansart 1974), et de l'autre des membres plus dotés en capitaux militants (Matonti \& Poupeau 2004: 8) qui tentent de valoriser des idées politiques largement désincarnées sous des formes de mobilisation partisanes plus traditionnelles.

\section{De jeunes intellectuel-le·s engagé·e·s tenté·e·s par la forme partidaire}

9 Avant de considérer les différentes prises de position des membres du RDR et les formes particulières de son militantisme, nous verrons d'abord qui sont les membres qui le composent en observant quelles dispositions variées ils sont susceptibles d'investir dans le groupe et pour quelles raisons leur engagement a pris une telle forme. Enfin nous verrons comment le RDR tente de se situer dans le sous-champ de la gauche radicale (Gottraux 1997), relativement à la Section française de l'internationale ouvrière (SFIO) et au Parti communiste français (PCF), mais aussi contre l'entreprise partisane gaulliste qu'est le Rassemblement du peuple français (RPF).

\subsection{Les membres du RDR : de jeunes intellectuel-le·s parisien $\cdot n e \cdot s$}

10 L'esquisse de sociographie du groupe, que nous souhaitons exposer en préalable de l'analyse des idées du RDR, se fonde sur une mise en série de données biographiques portant sur 1025 de ses membres. Comme souvent, nous avons été limité dans cette entreprise par le contenu modeste du fichier militant que nous sommes parvenu à reconstituer (Subileau $1981: 1039$ ) ; cette étude est toutefois complétée par les résultats d'une sociobiographie collective (Pudal \& Pennetier 2014 : 11) portant sur quatre-vingtdix-neuf militante's du RDR, dont les membres de son Comité directeur (CD). Suffisant à situer socialement les membres du RDR, ces données permettent de dire qu'ils sont très majoritairement des hommes (78,7\%), jeunes (l'âge médian est de 33 ans), habitant dans 
de grands centres urbains (au moins $60 \%)^{4}$. Ces tendances ne font pas exception au regard des autres organisations politiques de l'époque même si l'on constate que le recrutement du RDR est significativement plus jeune et plus urbain que celui de la SFIO (Rimbert 1955: 195), du MRP (Pépy 1955 : 209) ou du PCF (Collectif $1955: 181$ ). La distinction réside principalement dans la profession exercée puisque plus de $40 \% \mathrm{du}$ groupe exerce une profession intellectuelle. En effet, les professions libérales et cadres supérieurs, ainsi que les cadres moyens sont surreprésentés dans le Rassemblement, respectivement 5,5 fois et 4,2 fois plus nombreux que dans la population française ${ }^{5}$. Ces professions se distribuent entre les journalistes et instituteur.rice's $(16 \%$ des militant·es), les professeurees du secondaire et du supérieur, professions littéraires et scientifiques (9\%) et les avocate's et médecins (2,6\%). En outre, les étudiant·es représentent $7 \%$ du groupe militant. Dans la mesure où il peut être considéré comme le résultat d'un processus de promotion partisane (Pudal 1989:18) et de définition collective de l'image du groupe (Offerlé $1987: 107)$ ), la composition du Comité directeur (CD) dont plus de $82 \%$ des membres sont des intellectuelle's (douze journalistes, sept enseignante's, trois avocats, deux artistes ${ }^{6}$ ) participe à la construction de l'image du RDR comme "parti d'intellectuelle's ». Il ne faut cependant pas céder trop rapidement à cette qualification restrictive. D'abord parce que les positions intellectuelles occupées sont parfois très différentes: l'« intellectuel total » Jean-Paul Sartre (Bourdieu 1983) côtoie en effet le mathématicien Laurent Schwartz, l'avocat Jean Rous, le journaliste Georges Altman, l'instituteur Albert Demazière ou la professeure Henriette Morel', pour ne prendre que quelques exemples. Mais également parce que les dispositions qu'ils ont en commun et qu'ils tentent d'investir au moment de la construction d'un tel groupe ne renvoient pas uniquement à cette caractéristique.

11 La grande majorité a eu accès à une scolarisation avancée pour l'époque (13\% ont un certificat d'étude ou équivalent et plus d'un'e sur deux est diplôméee du supérieur). L'acquisition de capitaux scolaires rend alors possible, pour quelques rares cas, une trajectoire ascendante vers la petite-bourgeoise, et pour la majorité, le déplacement au sein de cette classe, vers les fractions valorisant la détention de capitaux culturels ${ }^{8}$. Précocement engagée's dans les organisations politiques de gauche, SFIO ou trotskistes, un grand nombre d'entre eux/elles disposent en 1948 d'un important capital militant mais également d'une certaine disposition à l'hétérodoxie politique. Nombreux sont ceux et celles, en effet, qui ont été excluee's ou ont quitté des groupes politiques au cours de leur trajectoire militante, ce qui leur confère un certain savoir-faire dans le militantisme minoritaire et souvent dans l'organisation et la mobilisation partisane. Enfin, il est utile de signaler que l'on connaît, pour la moitié d'entre eux/elles, une activité dans la Résistance. Dans le contexte de la fin des années 1940, une telle pratique est structurante, dans le champ politique (Dogan 1967:487) comme dans le champ intellectuel (Boschetti 2009). Sans surévaluer la croyance en une commune destinée des combattant-e's (Mariot $2017: 10)$ cette expérience partagée participe alors à la définition d'un capital symbolique que les militante's sont d'autant plus tentée'es de valoriser qu'il fait alors l'objet d'une déconsidération progressive (Laborie 2011). 
Fig. 1. Carte d'adhérent du R.D.R.

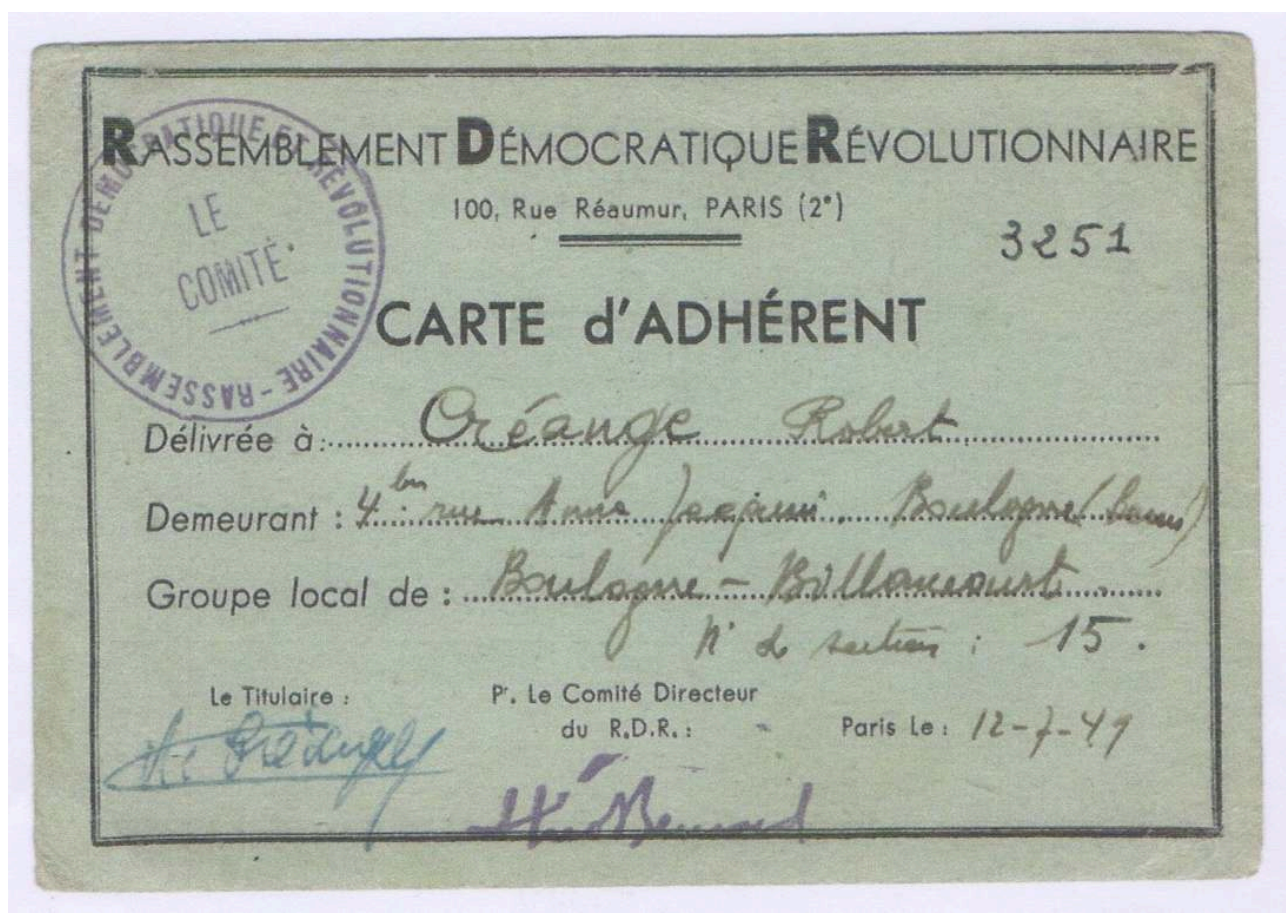

Carte d'adhérent n³251 attribuée le 12 juillet 1949 à Robert Créange

Source: collection particulière.

12 Si la construction d'un Rassemblement politique en 1948 peut apparaitre comme la tentative de valoriser l'engagement résistant face à la dynamique de dévaluation qu'il connait alors, c'est également le paradigme de l'intellectuel engagé qu'il s'agit de défendre. En effet, Sartre confère alors une responsabilité «écrasante » aux intellectuelle's (Sapiro 1999: 676), qui passe par la définition d'une figure de l'intellectuel qui prend position systématiquement (Sartre 2012 : 210) sur les enjeux de son époque. C'est dans une certaine mesure cette perspective d'une pratique intellectuelle engagée par nature que reprennent à leur compte les membres du Rassemblement. La forme de leur organisation est dans cette perspective un moyen de préserver la figure de l'intellectuel engagé, notamment face à la concurrence du PCF tant cette période est contraignante pour les intellectuelle's communistes, encouragé e's à mettre leur production au service du parti et à intégrer ce dernier dans des espaces militants dédiés, oblitérant dès lors toute autonomie de production (Sapiro 2018 : 178).

\subsection{Une entreprise partisane " entre déclin socialiste et repli communiste $^{9}$ »}

C'est d'abord une organisation politique de gauche que les membres du RDR entendent construire. En 1948, cela implique de faire exister une nouvelle position entre la SFIO qui participe alors au gouvernement de "troisième force " et le PCF revenu à une certaine forme d'opposition depuis mai 1947. Le titre de sa publication, La Gauche, qui paraît deux fois par mois à partir de la mi-mai 1948 est assez explicite. En référence à une expérience largement partagée par les membres du RDR, sa manchette se revendique d'«une nouvelle résistance internationale». La Gauche prétend à une 
diffusion nationale, au-delà des seul $\bullet \bullet$ s adhérent•e $\bullet$ s au RDR, et une grande part de sa fabrication et de son impression est effectuée par l'équipe du journal Franc-Tireur. L'implication de la rédaction du quotidien est non seulement visible dans les signatures des articles, mais aussi dans les sujets traités et dans l'orientation donnée à la publication. S'il s'agit bien d'un journal politique, considéré comme l'organe du RDR, le journal $\mathrm{La}$ Gauche traite également de l'actualité nationale et internationale et se situe sur le marché des publications politiques généralistes à diffusion nationale. Il relève de plusieurs formes distinctes de publications: à la fois organe du RDR et de sa vie militante, mais aussi journal d'actualité permettant d'exprimer le point de vue du Rassemblement sur de nombreux sujets, ou encore espace symbolique d'expression des intellectuelle's membres ou proches du RDR (comme c'est le cas de Claude Bourdet ou d'Albert Camus, membres de la rédaction de Combat). Cependant, La Gauche n'est diffusée en novembre 1948 qu'à 20000 exemplaires selon les Renseignements généraux ${ }^{10}$, sans que l'on puisse évaluer le nombre d'exemplaires payants effectivement vendus. On y retrouve cependant, à deux reprises au moins, l'évocation d'un «programme politique » du RDR ainsi que des éditoriaux publiés par les membres du RDR disposant d'une certaine notoriété : David Rousset, journaliste et écrivain, Georges Altman et Charles Ronsac, journalistes à Franc-Tireur ou encore Jean-Paul Sartre ${ }^{11}$.

Tout d'abord, David Rousset évoque l'existence d'un programme qui se résumerait à cette formule «redonner une base sociale vivante à la démocratie ». Cette intention passe par la mise en place des conditions d'un contrôle effectif de la gestion économique et de l'État par les travailleur-se's et les intellectuelle·s. Écartant les catégorisations disqualifiantes de "trotskistes" émise par Raymond Aron dans Le Figaro ou d'" aile gauche de la SFIO » prononcée dans l'Humanité, il réprouve également la qualification de "marxiste " pour ne revendiquer que l'héritage de la lutte durant l'Occupation. Aussi peut-on comprendre les formes de production d'idées par les dispositions de ces initiateurs, notamment acquises durant le combat résistant, qui les situent plus dans l'action que dans la définition d'un programme minutieux, comme le suggère Claude Bourdet: «la clandestinité, puis la prison avaient accentué chez la plupart de nous ce sentiment de l'effondrement certain de toute une société [...] mais cela ne conduisait pas à penser en plans et en schémas; les résistants avaient appris à compter sur la volonté active jointe à l'empirisme ; procédés peu français, mais qui ont fait leurs preuves ailleurs » (Bourdet 1952).

Présenté dans le troisième numéro de La Gauche, publié en juin 1948, le «Projet et plan de travail pour un programme du Rassemblement démocratique révolutionnaire " (voir encadré), permet cependant d'appréhender les prises de position du RDR sur la base de ce qui s'apparente à une ébauche de programme (RDR 1948). L'élaboration d'un tel texte permet aux membres du RDR de se conformer aux attentes du champ politique qu'ils entendent intégrer par la production d'une « norme de la transaction électorale » (Bué, Fertikh, Hauchecorne 2016: 10). Karim Fertikh a en effet montré combien la rédaction d'un tel texte gagnait à être réinscrite dans l'histoire d'une pratique politique devenue structurante qui est autant une prise de position politique synchronique qu'une « transaction avec une organisation et son passé » (Fertikh 2014 : 907). Dès lors, le RDR se conforme aux attentes du champ politique, ou les anticipe, dans le but d'y légitimer la construction d'une place nouvelle. Le collectif peut ainsi se prévaloir d'une prise de position unifiée qui est cependant le fruit d'accommodements entre ses diverses tendances (Andrieu 1984). Initiée par un préambule mettant en avant la 
supposée «trahison de la Résistance ", la présentation de ce programme débute par une tentative de clarification de la position du RDR au sein du champ politique de l'époque. Ainsi, les auteur.rice's se défendent de tout anti-communisme en indiquant qu'ils/elles se situent aux côtés du PCF, dans le «clan de la classe ouvrière », tout en regrettant le fait que les «mots d'ordres, les méthodes et les objectifs " censés servir ses intérêts seraient en réalité dictés au Parti par les exigences de "la politique extérieure soviétique ». Enfin, évoquant la " troisième force » comme une " coalition de conservatisme politique " à l'origine de la défection de nombreux militants révolutionnaires, le texte légitime la nécessité de construire le RDR pour tenter d'endiguer ce déclin, observable notamment par l'éclatement syndical.

Fig. 2. Jean-Paul Sartre

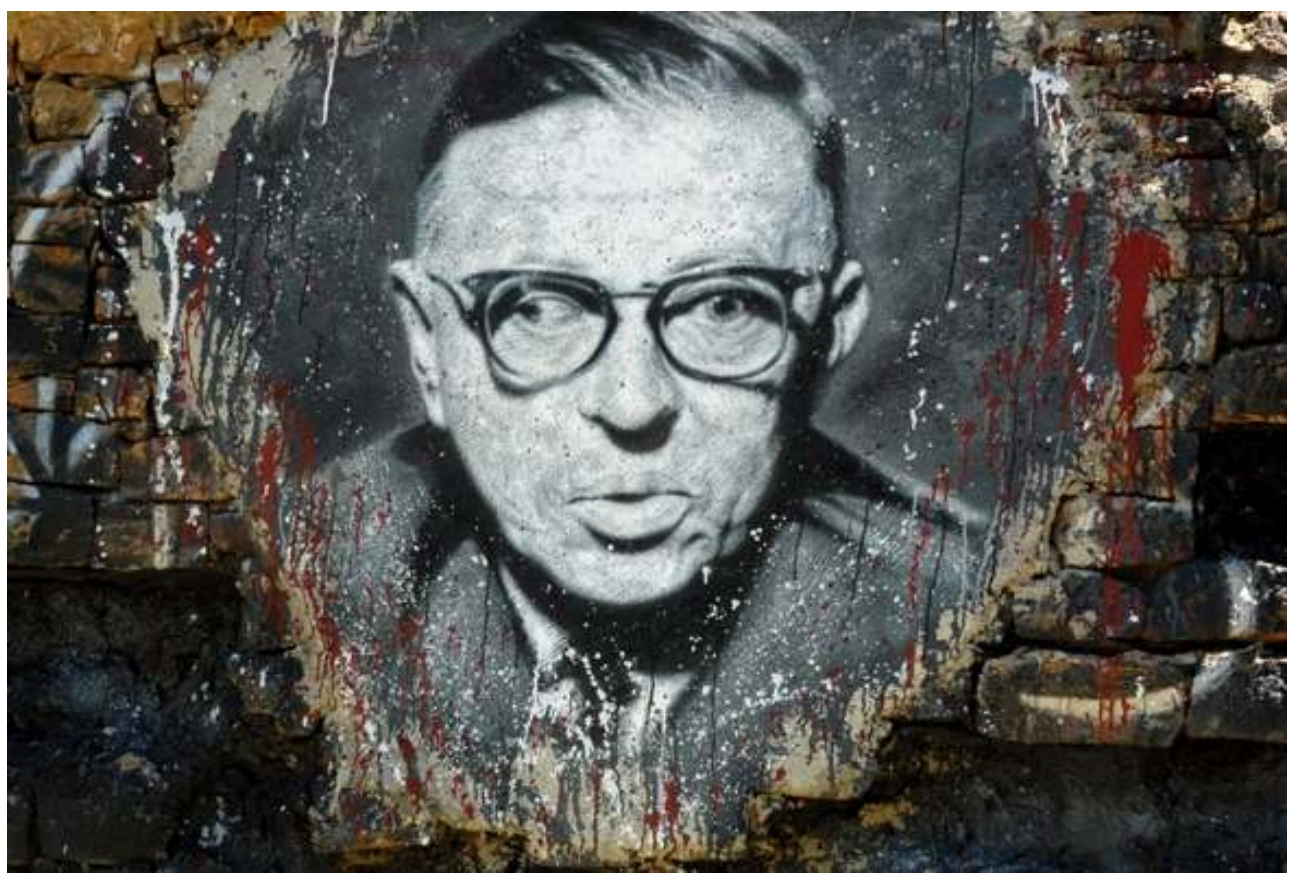

Thierry Ehrmann, « Jean-Paul Sartre », La Demeure du Chaos, musée d'art contemporain, SaintRomain-au-Mont-d'Or

Source: Licence CC BY 2.0; Flickr, 26 juin 2008.

16 Le « Projet et plan de travail pour un programme du Rassemblement démocratique révolutionnaire » est par la suite organisé en fonction de cinq points, correspondant à quatre niveaux d'intervention politique (le lieu de travail, le lieu de consommation, "dans les campagnes", en direction des jeunes et enfin concernant l'«Union française $»^{12}$.

"Projet et plan de travail pour un programme du Rassemblement démocratique révolutionnaire»

I. « Sur les lieux de travail » : création des « RDR d'entreprise » regroupant des travailleurs, quelle que soit leur appartenance partisane, syndicale ou confessionnelle pour le respect de la législation dans les entreprises et notamment le droit de grève, la constitution de l'échelle mobile des salaires, des comités d'entreprise permettant aux travailleurs d'empêcher les mécanismes visant à faire augmenter artificiellement les prix, notamment dans les entreprises produisant 
des biens de consommation de masse, la transformation de « la production de guerre en production de paix ", et enfin la " promotion ouvrière " par la formation professionnelle et culturelle des militants, permettant la préparation des ouvriers à la gestion directe de leurs entreprises. Par ailleurs, le RDR incite ses membres à se battre pour l'unité syndicale ${ }^{13}$.

II. « Sur le lieu de consommation »: les «RDR de quartiers et de communes » ont pour but d'organiser les membres du Rassemblement au niveau local comme consommateurs et usagers dirigés vers la lutte contre le marché noir et la hausse des prix. L'établissement et la promotion de circuits directs de consommation sont revendiqués, notamment au moyen de coopératives agricoles et de production ouvrière ainsi que la recherche de solution de logement pour les travailleurs qui en sont dépourvus. Enfin les militants sont enjoints à tenir des cahiers de revendications susceptibles d'organiser le contrôle permanent des représentants locaux, notamment dans les conseils municipaux.

III. « Dans les campagnes » : le RDR déclare soutenir les coopératives et syndicats paysans et « une alliance étroite des organisations démocratiques révolutionnaires des villes et des champs au sein d'organismes interprofessionnels comme au sein des RDR ruraux ». Le Rassemblement met notamment en avant la revendication de remembrement, de développement d'une industrie de production d'outillage agricole et d'engrais destinée aux petites exploitations, la généralisation de coopératives d'utilisation de matériel agricole ainsi que le « développement des circuits directs entre les campagnes et les villes par la suppression des intermédiaires parasitaires $"$.

IV. « Pour les jeunes » : les jeunes du RDR sont incités à investir les Maisons de jeunes, Auberges de la jeunesse, Cercles de la culture et centres d'apprentissage où ils peuvent notamment lutter contre l'augmentation de la durée du service militaire. Par ailleurs, le RDR propose plusieurs revendications relatives à l'éducation, notamment la défense de l'école publique laïque, le recrutement de maîtres « dignement rémunérés ", la réalisation de la réforme scolaire proposée par le projet Langevin, le développement de la gratuité par le moyen d'allocation de présalaire, la multiplication des centres de formation professionnelle et de centres de culture et de loisirs auxquels participeraient les enseignants et « l'accès des universités aux enfants du peuple ».

V. «Pour une Union française démocratique révolutionnaire » : le programme du RDR explicite l'opposition des membres du groupe aux « formes déguisées de l'ancien colonialisme, même abritées sous le nouveau statut de l'"Union française" ». Les auteur.rice's du texte exigent la fin de la guerre en Indochine et de la répression à Madagascar et revendiquent dans tous les territoires de l'Union française la liberté d'expression, d'association et d'auto-détermination.

(Source : RDR 1948.)

17 C'est donc bien au sein de la gauche du champ politique français que les membres du RDR prennent position, à la fois par leur programme économique et social, mais aussi par leurs tentatives de justifier leur légitimité à débattre avec les organisations concurrentes. Les doubles appartenances sont autorisées au sein du Rassemblement, 
qui espère ainsi créer un espace susceptible d'accueillir celles et ceux qui auraient été déçue's par les pratiques gouvernementales de la SFIO ou par les prises de position jugées restrictives du PCF. La faible institutionnalisation que permet la forme du rassemblement est par ailleurs à mettre en regard avec les positions de ses membres dans d'autres espaces. Que ce soit à la SFIO et au Parti communiste internationaliste (PCI - parti trotskiste), ou dans les rédactions de Franc-Tireur et des Temps Modernes, ils/ elles peuvent faire valoir leur participation au RDR dans les enjeux internes sans pour autant renoncer à une certaine indépendance vis-à-vis de leur nouvelle entreprise politique.

18 Le texte de « l'appel au Rassemblement démocratique révolutionnaire ${ }^{14}$ » publié le 28 février 1948 dans le journal Franc-Tireur, va dans ce sens. Les membres de ce comité d'initiative se définissent comme des « survivants de l'enfer, rescapés de la Résistance, militants, sympathisants ou compagnons de route des grands mouvements qui se réclament de l'émancipation sociale [...]» et invitent les militantees d'autres organisations ainsi que ceux et celles « qui ne se résignent pas à n'être nulle part et ne veulent point choisir» à se retrouver dans le «Rassemblement de la démocratie internationale et révolutionnaire». La nouvelle organisation est, dans une large mesure, conçue par ses instigateure's en opposition aux formes antérieures et contemporaines d'organisations politiques. La création du RDR relève d'une prise de position au sein du champ politique contre la SFIO tout d'abord, dont la participation aux coalitions gouvernementales traduirait «les pourrissements de la démocratie capitaliste et les tares d'une certaine social-démocratie ». Les rédacteur.ice's du texte critiquent par ailleurs l'oppression coloniale et "l'exaspération artificielle des nationalismes ». Le PCF, ensuite, est également dénoncé dans la mesure où il représenterait «la limitation du communisme à sa forme stalinienne». Le comité d'initiative estime que « la fin socialiste ne tolère pas l'utilisation de n'importe quel moyen [...] nous ne saurions admettre ce double jeu qui consiste à pratiquer, dans le présent, des moyens indignes, et que l'on sait tels, pour assurer, dans l'avenir, une fin noble ", ce qui rejoint une prise de position récurrente des organisations trotskistes contre le modèle stalinien (Bensaïd 2002:60).

19 Enfin, le cas de la profession de foi de Charles Marché à l'occasion des élections cantonales du 20 mars 1949 peut également être évoqué ${ }^{15}$. Candidat dans le canton de Niort 2, Charles Marché, ancien militant socialiste, produit et distribue l'une des rares prises de position du RDR sur le marché des biens électoraux. Il y fait référence à son engagement au sein de la SFIO et inscrit sa candidature dans sa continuité. Il reproche en revanche au parti socialiste son manque de cohérence : c'est ce dernier, en effet, selon Charles Marché, qui aurait trahi la classe ouvrière et la gauche en s'alliant avec le Mouvement républicain populaire (MRP - centriste) pour rester au gouvernement et en soutenant les écoles privées. Le texte met en avant la défense de la paix et de la laïcité et, dans la tradition des organisations revendiquant la représentation de la classe ouvrière, met en cause l'exploitation des travailleur'se's, mais aussi la bureaucratie.

20 Si c'est vis-à-vis de la SFIO et du PCF que le Rassemblement prétend principalement construire sa position, le contexte électoral rend nécessaire de ne pas négliger la droite alors partiellement rassemblée au sein du Rassemblement du peuple français (RPF fondé par le général de Gaulle). 


\subsection{Intellectuel collectif contre conseiller du prince : le Rassemblement de Sartre contre celui de Malraux}

21 En effet, la forme prise par le RDR n'est pas sans rappeler un autre pôle structurant le champ politique français à l'époque: celui de la droite en phase de recomposition autour du RPF. Créé en avril 1947, ce nouveau Rassemblement signe pour partie le retour des positions politiques conservatrices au sein du champ politique reconstitué après une phase durant laquelle, à la Libération, les organisations partisanes de gauches étaient quasi hégémoniques. Le programme du RDR déjà évoqué insiste d'ailleurs avant toute chose sur l'opposition au RPF en dénonçant « la fin des libertés démocratiques » et le « régime du parti unique » qui risquerait de découler de l'arrivée au pouvoir des gaullistes.

Comparer ces deux Rassemblements ne relève pas seulement d'un rapprochement typologique : les deux entreprises empruntent des directions souvent opposées malgré une démarche analogue. En effet, leur création s'inscrit dans un contexte de dévaluation des formes traditionnelles des partis politiques (comme en témoigne par exemple la critique gaulliste de «l'immobilisme » des notables radicaux - Fogacci 2015) et les deux rassemblements entendent marquer une distance avec les pratiques partisanes antérieures en autorisant la double appartenance et en revendiquant une institutionnalisation moins contraignante pour leurs membres. La recherche d'une certaine forme d'autonomie vis-à-vis des logiques internationales et nationales de distribution des positions politiques en fonction de l'alternative entre les blocs semble enfin rapprocher RPF et RDR. Cependant, c'est dans l'alternative qu'ils entendent proposer que les groupes s'opposent. En effet, au neutralisme anti-impérialiste du RDR répond une revendication d'autonomie de la France marquée par un anticommunisme virulent pour le RPF qui met la lutte contre les communistes, « séparatistes » supposés, au centre de ses logiques de mobilisation. Enfin, les logiques d'engagement intellectuel dont ils peuvent se prévaloir sont très différentes et se cristallisent notamment dans les figures engagées opposées de Jean-Paul Sartre et André Malraux.

La confrontation de Malraux avec le champ politique à la libération est fortement marquée par le statut de "conseiller du prince » qu'il acquiert dès le mois d'août 1945 lorsqu'il devient conseiller de Charles de Gaulle (alors président du Gouvernement provisoire de la République française), puis ministre de l'Information en novembre (Todd 2001: 380). S'il avait auparavant participé aux discussions, rapidement abandonnées, en vue de la construction d'une organisation politique unifiée issue de la Résistance, c'est également sur la base de cet engagement qu'il a débuté sa collaboration avec Charles de Gaulle (Lachaise 2009). À la différence de la perspective de l'engagement sartrien à cette époque (Boschetti 1985 : 258), la participation de Malraux à la fondation puis à l'activité politique du RPF est systématiquement liée à sa proximité individuelle avec la figure du général de Gaulle. Alors qu'il était parvenu avant-guerre à convertir un capital politique non partisan en capital littéraire (Sapiro 2018 : 343), il opère un retournement en 1947 en mettant sa notoriété littéraire (et résistante) au service d'une pratique partisane.

24 Sans revenir trop longuement sur la position atypique de Malraux à cette période, soulignons que celui-ci se trouve fortement isolé à gauche, entre le PCF, contre lequel il entend rapidement lutter politiquement, et les partisans d'une littérature engagée auxquels il oppose une pratique politique plus concrète, mais individuelle. La nécessité, 
pour lui, de préserver la reconnaissance associée à sa participation (largement réécrite) à la Résistance, puis aux combats (plus réels cette fois) de la Libération avec sa brigade Alsace-Lorraine (Todd 2001: 372), joue bien entendu dans la recherche d'une caution symbolique. Sartre, de son côté, même si son engagement résistant n'a pas été des plus avancés, peut bénéficier de la grande valeur symbolique associée à sa participation au Comité national des écrivains (CNE), institution collective de la Résistance littéraire qui détient à la Libération le monopole de la définition légitime de celle-ci (Sapiro 1999: 467). La figure du conseiller du prince incarnée par André Malraux s'oppose dès lors en tout point à la forme prise par « l'intellectuel collectif » RDR (Sapiro 2009 : 14). Quand la première réclame d'être positionnée parmi les dominants du champ littéraire, le second est plutôt le fait de dominées; quand le conseiller du prince offre un savoir généraliste à celui qu'il entend servir, l'intellectuel collectif rassemble des intellectuelle's située's au pôle autonome de leur espace d'origine (Bourdieu 2001 : 35). Si l'on considère l'intellectuel collectif comme mise en commun de savoir-faire, de ressources et de reconnaissances intellectuelles autrefois individuelles dans un collectif qui se donne pour principe une intervention dans l'espace public au nom de sa spécificité (Amiel 2017: 595 ; Pudal 2004: 185), on peut dès lors considérer que les militantees intellectuel-le's du RDR s'inscrivent, pour une partie au moins, dans une telle démarche. C'est au moins le cas de Sartre qui cherche, à un moment de consolidation de sa position dominante au sein du champ intellectuel, à inventer une position politique nouvelle susceptible de lui permettre de cumuler action politique concrète et autonomie intellectuelle (Amiel 2013 : 367).

\section{Formes d'intervention et prise de position du RDR : entre logiques partisanes et intellectuelles}

Après avoir esquissé le lien entre le recrutement du Rassemblement et les logiques d'engagement de ses membres en fonction de l'état du champ politique, nous pouvons nous intéresser à ses pratiques politiques et ses prises de position. Nous verrons alors dans un premier temps que les logiques partisanes et intellectuelles de mobilisation au sein du RDR s'inscrivent dans les formes mêmes de sa mobilisation. Par la suite, dans un contexte de forte injonction au positionnement en fonction de l'opposition «bloc atlantique » contre «bloc soviétique » nous observons que les prises de position du RDR font appel à la dimension internationale comme ressource hétérogène réinvestie dans les enjeux nationaux. Les revendications notables du groupe sont alors l'inscription dans le combat anticolonial et le refus de la guerre en devenir, ce qui passe par l'aspiration à une coalition européenne neutraliste.

\subsection{Formes intellectuelles d'engagement partisan}

Espace éditorial de formalisation des idées politiques du RDR les Entretiens sur la politique, publiés dans un premier temps dans Les Temps Modernes, la revue de Jean-Paul Sartre, puis sous la forme d'un ouvrage (Sartre, Rousset, Rosenthal 1949), augmenté d'un nouvel entretien avec Gérard Rosenthal, est le moyen de formaliser des prises de position et de toucher un public nouveau. Le choix de la publication dans la revue et en volume n'est pas anodin dans la mesure où le texte n'apparaît plus, dès lors, comme une simple production partisane, mais bien comme un livre bénéficiant de la valeur 
accordée à la sortie d'un texte inédit de Jean-Paul Sartre dans la " collection blanche " chez Gallimard, maison d'édition renommée. Les Entretiens s'inscrivent dans la continuité des publications de l'écrivain - même s'ils constituent un précédent dans la mesure où c'est son premier ouvrage non individuel - et sont donc susceptibles d'être achetés et lus par un public qui n'a pas la politique pour première logique d'achat ou de lecture ${ }^{16}$. Il s'agit d'une forme de production d'idées partisanes dans un espace éditorial autonome qu'ils peuvent donc espérer rendre audible au sein du champ intellectuel (Matonti 1996 : 95).

Les auteurs se concentrent dans un premier temps sur la justification de la création de leur collectif qui vise, sous la forme d'un rassemblement plutôt que d'un parti, à agréger la classe ouvrière et la classe moyenne dans une organisation gérée démocratiquement par ses membres et à «briser " ainsi la "scholastique marxiste " (Sartre, Rousset, Rosenthal 1949: 12), comme le préconise David Rousset. Ainsi, les membres du RDR convoquent le modèle du RPF comme moyen partisan efficace d'unification des fractions de la classe bourgeoise tout en dénonçant ses idées réactionnaires et son mode d'organisation. Selon Jean-Paul Sartre, « il est bien évident que le RPF table sur l'idée de la France éternelle, et, de ce point de vue, il est certain que la politique qu'il peut adopter, soit sur le plan international, soit sur un plan national, n'est au fond qu'une politique opportuniste et épisodique destinée à sauver certains éléments d'une France prétendue invariable» (Sartre, Rousset, Rosenthal 1949: 37-38). Et David Rousset de renchérir sur l'absence de programme social du Rassemblement gaulliste, qu'il explique par la récupération de « la conception vichyste et donc, à mon sens, la conception la plus réactionnaire en France » (Sartre, Rousset, Rosenthal 1949 : 57). 
Fig. 3. Entretiens sur la politique

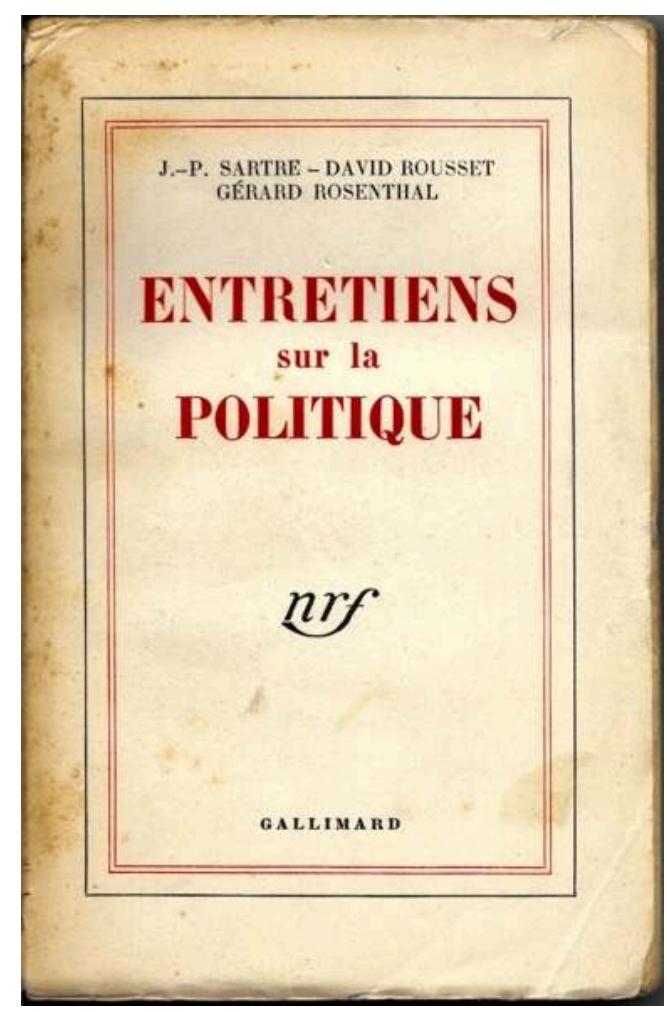

Couverture de l'ouvrage Entretiens sur la politique, par Jean-Paul Sartre, David Rousset et Gérard Rosenthal (Gallimard, 1949)

Source: Collection personnelle

On retrouve cette opposition dans les prises de paroles lors d'un «meeting d'intellectuels » organisé par le RDR (Bernard 1949) - meeting dont l'appellation, que l'on retrouve également chez les Renseignements généraux, traduit bien la double allégeance, intellectuelle et partisane. Celui-ci, intitulé « L'internationalisme de l'esprit et la paix dans la monde ", s'est tenu à Paris, salle Pleyel, le 13 décembre 1948. Cet événement donne un bon exemple de production de prises de position au sein du RDR, sous forme des discours publics d'intellectuelle-s dans un contexte de recomposition de l'espace intellectuel (Boschetti 2009) qui met en jeu la définition sartrienne de l'intellectuel engagé (Sapiro $2018: 73$ ).

En effet, outre les membres du Rassemblement, Georges Altman, Gérard Rosenthal, David Rousset et Jean-Paul Sartre, d'autres personnalités présentes ont la particularité d'occuper des positions politiques proches du RDR, sans avoir investi cette entreprise au-delà d'un soutien symbolique : c'est le cas d'Albert Camus, André Breton, Claude Bourdet et Simone de Beauvoir. Ces dernier e's côtoient de "grands écrivains d'Europe et d'Outre-mer" comme le proclame l'affiche annonçant l'événement. Ces intellectuelle's sympathisante-s contribuent à la mobilisation politique du RDR à travers des interventions qui ont pour principe leur position d'intellectuelle's et le capital symbolique qui y est rattaché. La formule utilisée par l'intellectuel vietnamien Le Quan-Dung, «cette puissance supérieure à toutes: l'esprit» (Quan-Dung 1948), résume bien leur prétention à faire valoir des modalités d'action politique concurrentes des formes traditionnelles de militantisme pour garantir la paix, et l'intégration de 
leurs discours dans un processus performatif qui consiste à croire dans leur capacité mobilisatrice.

Devant deux-mille-cinq-cents personnes selon la police ${ }^{17}$, et plus de cinq-mille selon les organisateurs (Altman 1948), se succèdent notamment les interventions de Richard Wright, écrivain nord-américain, notamment auteur des romans Native Son et Black Boy ( Wright 1947a; 1947b) ancien adhérent du parti communiste et membre de la rédaction de la revue Les Temps Modernes; Jef Last, écrivain, poète et sinologue hollandais, ami et traducteur d'André Gide, ancien militant du parti socialiste néerlandais, du parti communiste et membre des brigades internationales (Gide \& Last 1985); ou encore Juan Andrade, journaliste et éditeur espagnol, militant du Parti ouvrier d'unification marxiste (POUM) créé à Barcelone en 1935 et engagé dans la guerre civile espagnole, et ancien membre fondateur du parti communiste espagnol. Le meeting est ici organisé pour faire nombre, d'autant plus que la description de la foule amoncelée sur les trottoirs est alors un indicateur systématique de la réussite d'un meeting politique (Cossart $2010: 241$ ).

31 Ces nombreux soutiens internationaux, extérieurs au Rassemblement, acceptent de mettre leur légitimité littéraire et intellectuelle à son service, dans une logique de prise de position politique, notamment dans la mesure où leur position au sein de leur champ intellectuel national d'origine est régulièrement fonction de la valorisation de la dimension politique de leur littérature. La présentation de la journée faite par le journal Franc-Tireur est d'ailleurs l'occasion de rappeler les dimensions politiques de leurs œuvres et renvoie à une logique de "mise en texte " du meeting après son déroulement (Cossart $2001: 136)$ :

"C'est un événement non point seulement parisien, mais qui dépasse les frontières que cette grande confrontation publique des représentants de la culture mondiale : invités par le Rassemblement démocratique révolutionnaire, ils viennent ce soir à 20h30, salle Pleyel, dans la liberté totale de leur expression, appeler à l'" internationalisme de l'esprit ". Qui sont-ils ? Vous les connaissez déjà. Il y aura André Breton, le grand écrivain et poète rebelle; il y aura Albert Camus dont on aime l'âpre, l'intransigeante dignité; David Rousset, puissant témoin du monde concentrationnaire et magnifique tribun; Jean-Paul Sartre, dont l'intelligence si humaine, le talent aigu, la profonde honnêteté intellectuelle sont appréciés d'un large public, Simone de Beauvoir, essayiste et romancière de grand talent. Richard Wright, dont on a lu les admirables romans sur la vie des noirs aux États-Unis, " Native Son ", "Black Boy" ; Carlo Levi qui, par son livre, " Le Christ s'est arrêté à Éboli", apporte un poignant témoignage sur la vie des Italiens antifascistes en Italie même. [...] Pour la première fois également, paraitra devant le peuple démocratique et révolutionnaire de Paris Théodor Plievier, le grand écrivain allemand antinazi, l'auteur de ce livre foudroyant, " Stalingrad ", dont la traduction en français fait déjà sensation à Paris [...] Une grande rencontre, en vérité, où le talent, l'originalité, l'art de chacun de ces grands intellectuels fera prendre à la lutte pour la liberté et pour la fraternité une résonance profonde et des accents nouveaux ${ }^{18}$ ».

32 À contre-courant du processus, mis au jour par Paula Cossart, qui consiste pour la presse partisane à privilégier la publication de photographies du public dans la perspective d'une mise en spectacle du nombre, le numéro que La Gauche consacre au meeting ne se préoccupe pas de mettre en image la foule et intègre en revanche des portraits des orateurs et de l'oratrice, qui sont ainsi valorisée's individuellement comme intellectuelle's. Pour la plupart, les interventions sont cantonnées au registre de la prise de position surplombante d'intellectuelle’s vis-à-vis des enjeux politiques et 
plus particulièrement de la guerre en devenir, qui est au centre de leurs préoccupations.

Dénonçant les partis politiques comme incapables d'empêcher un tel conflit revendiquant par là une certaine autonomie intellectuelle vis-à-vis des organisation partisanes - mais aussi la recrudescence des prises de position nationalistes, André Breton livre ainsi un discours visant à contester le conflit putatif: " c'est ce nationalisme ivre et encore avide de sang qu'aujourd'hui nous devons juguler où qu'il se trouve, c'est cet impérialisme rival du coca-cola et du marxisme dénaturé que nous devons, par les voies les plus promptes, mettre hors d'état de consommer le sacrifice de nos vies ». Dans son intervention, Jean-Paul Sartre prône la construction d'une Europe socialiste contre le totalitarisme "sous l'une ou l'autre de ses formes ", mais pose en préalable la démilitarisation des pays et des esprits.

Cet appel aux intellectuellle's émerge dans la plupart des prises de paroles. De fait, bien qu'appuyé sur le RDR, le « meeting d'intellectuels » reste une réunion s'adressant à des intellectuels. David Rousset entend ainsi mobiliser ces dernier·e's au nom de l'unité de la liberté qui, « si elle cesse d'exister dans le domaine de la politique, (...) cesse d'exister dans le domaine des arts et des sciences " avant de faire référence à l'existence de camps de concentration en URSS qu'il dénonce.

L'intervention de Richard Wright permet de saisir plus explicitement encore l'intention des discours et le public visé : il s'agit d'intellectuel-le·s s'adressant à leurs pairs. Wright dénonce en effet la guerre froide non comme un conflit opposant l'URSS aux États-Unis, mais comme une guerre contre la culture. Il met sur un pied d'égalité Hollywood et Prague : « écoutez écrivains et artistes : les hommes qui, aujourd'hui, mènent le monde vous ont déclaré la guerre!», prévient-il. Il préconise un engagement politique en redéfinissant, à la suite de Sartre, la dichotomie entre actes et discours, "des actes, voilà ce que vous, intellectuels, devez accomplir, des actes avec des mots, des actes qu'exprimeront vos besoins, vos désirs, vos rêves ".

\subsection{Prise de position politique : lutte anticoloniale et construction d'une alternative européenne aux « blocs »}

La position du Rassemblement concernant la politique internationale se fonde sur le constat suivant : "trois ans après la fin des hostilités, les deux plus grandes puissances sont aux prises dans une "guerre froide" dont chaque épisode accroît de part et d'autre la crainte d'une nouvelle guerre mondiale plus dévastatrice que jamais » (RDR 1948). Dès lors, entrer dans le jeu des blocs reviendrait à précipiter le conflit dans lequel les États-Unis tentent de maintenir le capitalisme pour assurer leur hégémonie économique, quitte à soutenir n'importe quel régime empêchant l'extension de l'URSS, y compris des dictatures, tandis que l'URSS, qui considère que le socialisme est la solution pour la paix, base son développement sur l'extension du modèle russe « qui ne respecte pas toutes les libertés ». Ainsi, afin de ne pas risquer d'avoir l'air de soutenir l'un par la dénonciation de l'autre, les membres du RDR refusent de choisir et préconisent «la paix par et pour la démocratie révolutionnaire ». Ils refusent par ailleurs la participation de la France à des ensembles économiques ou politiques qui la situeraient dans l'un des blocs, et revendiquent un « bouleversement révolutionnaire et démocratique des structures nationales" au sein d'une Fédération des peuples européens. Les auteurs du plan de travail du RDR appellent à une lutte constructive 
contre le plan Marshall, présenté à la fois comme nécessaire et problématique. Enfin, le texte contient un appel à la lutte contre la militarisation du plan et exige un contrôle syndical de la répartition et de l'utilisation des aides afin notamment de l'ouvrir au commerce avec le bloc de l'Est.

L'opposition entre les blocs participe à une dynamique de guerre froide qui structure partiellement les oppositions politiques en fonction de l'alternative «Est-Ouest » et de l'injonction au positionnement qui pèse sur les individus et les collectifs. Cependant, l'originalité du RDR réside dans le refus de se positionner, Jean-Paul Sartre précise ainsi: "en fait nous ne sommes contre personne. Un fatalisme de la guerre est aujourd'hui répandu en Europe, qui fait que les uns, cherchant une protection du côté des USA, se trouvent nécessairement prendre parti contre l'URSS, comme on le voit par l'exemple du RPF, et que d'autres, cherchant une protection contre le fascisme ou contre le capitalisme impérialiste des USA, se jettent dans les bras de l'URSS et deviennent, eux aussi, des facteurs de guerre. Il nous a paru que l'existence de ces deux camps qui caractérise l'Europe d'aujourd'hui est née de la division du monde en deux groupes de puissances rivales et devient par un choc en retour une cause de guerre " (Sartre, Rousset, Rosenthal 1949 : 82). Résister, au nom d'une position intellectuelle - la défense de la paix -, à une alternative à un moment où cette dernière structure fortement les enjeux nationaux et internationaux est le moyen pour les membres $d u$ RDR de faire valoir (sans succès) dans un espace français concurrentiel une ressource distinctive produite en fonction d'enjeux internationaux, mais aussi de revendiquer une traduction politique de leurs ressources intellectuelles.

Ces prises de position rejoignent celles émises par les fondateurs et fondatrices du Mouvement socialiste pour les États-Unis d'Europe, mouvement situé au "pôle politique " de l'espace relationnel transnational européen en voie de structuration (Cohen 2006 : 116), auquel participent le RDR et ses membres, aux côtés de la SFIO et de personnalités comme Georges Izard ou Claude Bourdet. Cela contribue à conférer au Rassemblement une certaine légitimité à la fois au sein du champ politique français, mais aussi au niveau transnational, ce qui représente une ressource mobilisable de façon ponctuelle et locale (Gobille 2005). De fait, la théorisation d'une Europe "neutre » facteur de paix entre les blocs est par ailleurs pour partie inspirée de l'exemple indien et de la politique menée par son premier ministre, Nehru, après l'indépendance qui conduira par la suite à la définition de l'alliance internationale des « non-alignés » (Lee 2010 ; Durantin 1981).

Finalement, c'est autour de ce refus que s'articulent les revendications sociales du RDR : "le RDR est un rassemblement démocratique et révolutionnaire; ce qui lui dictera son attitude dans l'appréciation en chaque cas des chances internationales de la démocratie et de la révolution. Contre toute occupation de caractère oppressif, qu'elle vienne de l'URSS ou des USA., il se constituera comme organe clandestin de résistance. [...] En conséquence, avant de régler sa politique sur le devenir d'un conflit mondial qui ne laisse aucun espoir à la démocratie, le RDR se préoccupe d'abord de manifester inconditionnellement contre la guerre. Et il n'y a pas de lutte contre la guerre qui ne soit pas une lutte sociale » (Sartre, Rousset, Rosenthal 1949 : 183-185).

40 Déjà évoquée dans son Appel, la lutte contre l'oppression coloniale fait partie des prises de positions du groupe. En effet, le collectif reproche à la SFIO la répression des soulèvements malgaches et vietnamiens, ainsi que les procès intentés aux militants indépendantistes. Si les positions peuvent diverger partiellement entre les membres du 
groupe quant à la forme immédiate prise par l'émancipation des peuples colonisés (autonomie ou indépendance) cette prise de position est revendiquée comme l'une des plus distinctives du RDR. Il faut également rechercher dans les trajectoires antérieures de ses membres les raisons d'un tel engagement. Militantees à Paris dans les années 1920-1930, alors que la ville était le lieu de définition collectif d'un « nationalisme antiimpérialiste " (Goebel $2017: 7-8$ ), ils/elles ont très tôt pris position pour la défense des peuples colonisés. Dans les Entretiens sur la politique, David Rousset propose une "autonomie politique, administrative et juridique» aux territoires d'outre-mer et d'Afrique du Nord, afin de créer des partenariats économiques profitant de leurs ressources. Il précise plus tard que, selon lui, le gouvernement s'apprête à lancer une action militaire au Viêt-Nam, et dénonce un procès scandaleux à Madagascar ayant dressé la population malgache contre la France. « Le gouvernement poursuit en Afrique du Nord une politique tatillonne, imbécile, policière, à l'égard d'éléments comme Messali Hadj qui représente un parti important de l'Algérie, ajoute-t-il, au Maroc on assiste à une politique de censure et d'asphyxie $»^{19}$. Il précise en guise de conclusion, « je suis délibérément pour accorder aux peuples des territoires d'outre-mer le droit à l'indépendance » (Sartre, Rousset, Rosenthal 1949 : 191). Gérard Rosenthal pense quant à lui qu'il faut permettre à tout moment à ces territoires de prendre leur indépendance, mais que les travailleurs français et de ces territoires ont plus intérêt à la mise en place d'un concordat regroupant les diverses entités. Originale dans le champ politique de l'époque, cette position est notamment relayée auprès d'organisations de lutte contre la politique coloniale comme le Congrès des peuples, auquels appartiennent des membres du RDR tels que Jean Rous.

Intervenir en tant qu'organisation politique contre la constitution de l'Union française ne revient pas seulement pour le RDR à continuer les luttes antérieures pour l'indépendance des peuples colonisés. Il s'agit aussi d'un moyen de contester l'ordre bipolaire en cours d'établissement, en nouant des alliances avec des mouvements révolutionnaires de lutte pour l'indépendance. De tels discours, émis durant une période de conflit en Indochine et de répression à Madagascar, reviennent à tenter de faire exister une position originale, tant sur le plan national qu'international par le biais en particulier d'interventions intellectuelles. Ainsi des militants vietnamiens, malgaches ou algériens sont invités à participer aux meetings d'intellectuelle's organisés par le RDR; des militants comme Messali Hadj, leader du parti du peuple algérien, sont interrogés dans $L a$ Gauche qui consacre par ailleurs des articles aux mouvements palestinien, algérien, indochinois ou malgache. À la fin des années 1940 une telle position est rare et contraste notamment avec les partis de la gauche gouvernementale (Biondi \& Morin1992: 286), faisant du RDR un précédent dans l'histoire longue d'une gauche non communiste minoritaire située aux côtés des peuples colonisés (Hamon \& Rottman 1979 : 36).

En somme, qu'elles prennent la forme d'un refus systématique de répondre à la "rhétorique du choix» ou celle de discours surplombant dénonçant une possible troisième guerre mondiale, les idées politiques du RDR apparaissent comme originales dans le champ politique français. Cependant, la construction d'une alternative passe par la coalition $d u$ RDR avec d'autres organisations, françaises et étrangères, notamment autour d'un projet de Mouvement socialiste pour des États-Unis d'Europe. Néanmoins, la dualité des pratiques au sein du Rassemblement, due à l'impossible croisement entre les logiques intellectuelles et partisanes de mobilisation (Matonti 2005b ; Sapiro 2009) rend partiellement inaudibles ces positions. C'est d'autant plus le 
cas que les membres intellectuels de l'entreprise politique limitent leur engagement à la production de discours largement déconnectés de la mobilisation partisane du RDR.

On trouve dans la confrontation de ces logiques différenciées de mobilisation, l'un des facteurs explicatifs de la fin rapide des activités du groupe. Les logiques intellectuelles et politiques qui devaient se compléter dans la perspective d'un engagement «total» (Bourdieu 1983) ne sont pas suffisamment intégrées dans les pratiques partisanes concrètes et les effets de champ auxquels les membres entendaient échapper par la construction d'un collectif dépassant les frontières finissent par s'imposer à eux et à disqualifier leur tentative dans l'un et l'autre des espaces. Les mobilisations intellectuelles du RDR ont quelque chose d'incantatoire et ses membres semblent difficilement percevoir, pour celles et ceux qui sont les plus éloignée's des pratiques partisanes, le coût et les efforts de mobilisation concrets nécessaires à la pérennisation d'une entreprise partisane.

\section{Conclusion. La rencontre manquée de l'intellectuel collectif et de l'entreprise partisane}

L'analyse de la production idéelle des membres du RDR permet de saisir la particularité de cette entreprise politique inscrite au sein de la gauche du champ politique français dans un contexte de guerre froide. La recherche d'une position originale au sein du sous-champ de la gauche, entre la SFIO et le PCF, ainsi que les prises de position hétérodoxes du point de vue de la lutte anticoloniale et de l'affrontement entre les blocs, tiennent aux dispositions particulières et hétérogènes de ses membres (notamment des intellectuelle's disposant d'un certain volume de «capital réputationnel »). S'il est ainsi possible de déceler la concentration de la production des idées dans des espaces qui ne sont pas au centre de l'appareil de mobilisation partisane, c'est aussi à cause de la coexistence en son sein de militante's disposant exclusivement d'un capital politique, et d'autres qui se caractérisent par leur position d'intellectuel-le's.

Finalement, ce qui se dégage de la place des idées dans ce « parti d'intellectuelle·s » est l'usage d'un nombre important de sources de production idéologiques exogènes, $y$ compris au sein du champ de production idéologique (Bourdieu 1979: 465). À l'intérieur du groupe, il existe une structuration particulière de l'activité politique, séparant la production de discours politiques relevant des "idées" - réservée à des espaces intellectuels "autonomes»- et les espaces de mobilisations partisanes tels que les comités locaux, les réunions publiques et les opérations électorales, pratiques politiques militantes moins impliquées dans la production de discours. Tout se passe comme si coexistaient deux espaces partiellement étanches : un intellectuel collectif (Pudal 2007) et une organisation partisane. Ici considéré comme regroupement d'intellectuelless tirant leur légitimité à intervenir politiquement, à la fois de l'autonomie de leur espace d'origine et des connaissances spécifiques accumulées dans leur domaine, l'intellectuel collectif a pour principe de mettre au service d'une prise de position politique, des ressources intellectuelles et symboliques forgées et utilisées par ses membres. En l'occurrence, et malgré les positions dominantes occupées par certain'es intellectuelle's du Rassemblement, son inscription légitime et durable au sein du champ politique est un échec à la fois par la disqualification du rôle politique des intellectuelle's à l'époque, mais également par un rapide repli du champ politique 
vers des formes partisanes traditionnelles. De l'éclatement de l'espace de production idéelle au sein du RDR découle pour partie une division du travail politique qui tend non seulement à réserver la production d'idées aux intellectuelle·s, mais aussi à les cantonner dans des espaces proches de leurs habitudes professionnelles, cloisonnant d'autant les répertoires de l'action collective dont le RDR fait un usage discrétionnaire. La «rencontre» espérée et revendiquée entre ces deux formes de mobilisation n'aboutit finalement pas, notamment parce que la structure du groupe ne suffit pas à remettre en cause les frontières symboliques entre les pratiques des militantees associées à leurs habitus, notamment acquis au sein de leur champ d'origine - le champ politique pour les militante's disposant d'un certain volume de capital politique, et le champ intellectuel pour celles et ceux qui ont fait profession de la production de biens symboliques. Persiste ainsi, au sein du groupe, une dichotomie entre les militantees engagée's dans des pratiques partisanes concrètes, et les militant·es intellectuelle's auxquellle's la croyance dans la performativité de leurs discours interdit de mettre en place les dispositifs nécessaires à leur déclinaison dans une mobilisation partisane plus tangible.

\section{BIBLIOGRAPHIE}

AGRIKOLIANSKY Éric (1997). La Ligue des droits de l'homme (1947-1990). Pérennisation et transformations d'une entreprise de défense des causes civiques. Thèse de doctorat en science politique. Paris, Institut d'études politiques de Paris.

ALTMAN Georges (1948). « Nous étions cinq milles... ». La Gauche, 10.

AMIEL Bastien, SEMPÉ Mathilde, DIRKX Paul (2015). «Crises politiques et champs littéraires : un objet interdisciplinaire ». COnTEXTES, 16. [Consulté le 01 avril 2021].

AMIEL Bastien (2013). « Sartre et le RDR : inventer une position politique ». In PAGÈs Claire et schumm Marion (dir.). Situations de Sartre. Paris, Hermann : 345-358.

AMIEL Bastien (2017). La Tentation partisane. Le Rassemblement démocratique révolutionnaire : une entreprise politique en construction entre «Libération » et " guerre froide ». Thèse de doctorat en science politique. Nanterre, Université Paris Nanterre.

ANSART Pierre (1974). Les Idéologies politiques. Paris, Presses universitaires de France.

AZAM Nicolas (2019). « Entre "croyance économique” et contrainte partisane. Genèse et structuration de la section économique du PCF (1947-1961)». Politix, 126 : 37-60.

BANDIER Norbert (1999). Sociologie du surréalisme. 1924-1929. Paris, La Dispute.

BARTHAS Jérémie \& SKORNICKI Arnault (2017). « Idées, histoire et sciences sociales. Entretiens avec Quentin Skinner ». In SKORNICKI Arnault \& GABORIAUX Chloé (dir.) (2017). Vers une histoire sociale des idées politiques. Villeneuve-d'Ascq, Presses universitaires du Septentrion : 93-110.

BELORGEY Nicolas, CHATEIGNER Frédéric, HAUCHECORNE Mathieu, PÉNISSAT Étienne (2011). « Théories en milieu militant. Introduction ». Sociétés contemporaines, 81 :5-25. 
BENSAID Daniel (2002). Les Trotskysmes. Paris. Presses universitaires de France, coll. « Que sais-je ». BERNARD Théo (1949). « Rapport d'organisation ». Bulletin intérieur, 1, ronéotypé, février : 20. BIONDI Jean-Pierre \& MORIN Gilles (1992). Les Anticolonialistes (1881-1962). Paris, Robert Laffont. BIRCHALL Ian (1999). « Neither Washington nor Moscow ? The Rise and Fall of the Rassemblement Démocratique Révolutionnaire ». Journal of European Studies, 24 : 365-404.

BOSCHETTI Anna (1985). Sartre et « Les Temps Modernes». Une entreprise intellectuelle. Paris, Minuit. BOSCHETTI Anna (2009). « La Recomposition de l'espace intellectuel en Europe après 1945 ». In SAPIRO Gisèle (dir.). L'Espace intellectuel en Europe. De la formation des États-Nations à la mondialisation, $X I X^{e}-X X I^{e}$ siècle. Paris, La Découverte : 147-182. BOURDET Claude (1952). L'Observateur, 21 août. BOURDIEU Pierre (1977). «La Production de la croyance. Contribution à une économie des biens symboliques ». Actes de la recherche en sciences sociales, $13: 3-43$.

BOURDIEU Pierre (1979). La Distinction. Critique sociale du jugement. Paris, Minuit.

BOURDIEU Pierre (1983). « Sartre, l'invention de l'intellectuel total ». Libération, 31 mars. Repris dans BoURDiEu Pierre (2002). « Sartre, l'invention de l'intellectuel total ». Agone, 26/27 : 225-226. BOURDIEU Pierre (2001). Contre-feux 2. Pour un mouvement social européen. Paris, Raison d'Agir. BRUN Éric (2014). Les Situationnistes. Une avant-garde totale (1950-1972). Paris, CNRS Éditions. BUÉ Nicolas, FERTIKH Karim, HAUCHECORNE Mathieu (2016). « Introduction. Les programmes politiques dans une perspective sociohistorique ». In BUÉ Nicolas, FERTIKH Karim, HAUCHECORNE Mathieu (dir.). Les Programmes politiques. Genèses et usages. Rennes, Presse universitaires de Rennes : 9-22.

CASEVITZ Jean (1947). « Le Recensement du 10 mars 1946 ». L'Information géographique. 11 (3) : 102-111.

CHARLE Christophe (1990). Naissance des « intellectuels », 1880-1900. Paris, Minuit. CHARLE Christophe \& JEANPIERRE Laurent (dir.) (2016). La Vie intellectuelle en France. Tome I, des lendemains de la Révolution à 1914 ; Tome II, de 1914 à nos jours. Paris, Seuil.

CLERC Paul (1964). « Changement dans la structure socio-professionnelle de la France entre 1954 et $1962 »$. Population, 19(4):683-706.

COHEN Antonin (2006). « De congrès en assemblées. La structuration de l'espace politique transnational européen au lendemain de la guerre ». Politiques européennes, 18 : 105-125.

COLLECTIF (1955). « Le Parti communiste français ». In DUVERGER Maurice (dir.). Partis politiques et classes sociales en France. Cahiers de la Fondation nationale des sciences politiques, Série « Partis et Élections ", 74. Paris, Armand Colin : 181-193.

cos Rafaël (2019). « La Carrière de la "sécurité” en milieu socialiste (1993-2012). Sociologie d'une conversion partisane ». Politix, $126: 135-161$.

COSSART Paula (2001). « La Communion militante. Les meetings de gauche durant les années trente ». Sociétés et Représentations, 2(12) : 131-141.

COSSART Paula (2010). Le Meeting politique. De la délibération à la manifestation (1868-1939). Rennes, Presses universitaires de Rennes. 
DOBRY Michel (1992) [1986]. Sociologie des crises politiques. La dynamique des mobilisations multisectorielles. Paris, Presses de la Fondation nationale des sciences politiques.

DOGAN Mattei (1967). «Les Filières de la carrière politique en France ». Revue française de sociologie, $8(4): 468-492$.

DULONG Delphine (2010). La Construction du champ politique. Rennes, Presses universitaires de Rennes.

DURANTIN Jean-François (1981). Le Problème du neutralisme en France de 1947 à 1953. Mémoire de maitrise d'histoire. Paris, Université Paris 1 Panthéon-Sorbonne.

FAUCONNIER Clémentine (2019). « La Mise en politique sans la mise en parti ? Le Centre de la politique sociale conservatrice comme entreprise doctrinale ». Politix, $126: 61-83$.

FERTIKH Karim (2014). « Le Genre programmatique. Sociologie de la production des programmes partisans : l'exemple de Bad Godesberg ». Revue française de science politique, 64(5) : 903-916.

FOGACCI Frédéric (2015). «Le monde de la notabilité radicale et radicale-socialiste de l'après-guerre : entre survie et adaptation (1945-1969)». Histoire@politique. Politique, culture, société, 25. [Consulté le 01 avril 2021].

FULLA Mathieu (2019). « Le Parti, l'expert et les théories économiques. Le cas du parti socialiste d'Épinay (1971-1981)». Politix, $126:$ 85-109.

GAXIE Daniel (2003) [1993]. La Démocratie représentative. Paris, Montchrestien.

GEAY Bertrand (1999). Profession : Instituteurs. Mémoire politique et action syndicale. Paris, Seuil. GIDE André \& LAST Jef (1985). Correspondances : 1934-1950. Lyon, Presses universitaires de Lyon. GOBILLE Boris (2005). « Les Altermondialistes : des activistes transnationaux? ». Critique internationale, $27: 131-145$.

GOBILLE Boris (2008). Mai 68. Paris, La Découverte, coll. « Repères ».

GOEBEL Michael (2017). Paris, capitale du tiers monde. Comment est née la révolution anticoloniale (1919-1939). Paris, La Découverte.

GOTTRAUX Philippe (1997). Socialisme ou Barbarie. Un engagement politique et intellectuel dans la France de l'après-guerre. Lausanne, Payot.

GOUARNÉ Isabelle (2013). L'Introduction du marxisme en France. Philosoviétisme et sciences humaines. 1920-1939. Rennes, Presses universitaires de Rennes.

HAMON Hervé \& ROTMAN Patrick (1979). Les Porteurs de valises. La Résistance française à la guerre d'Algérie. Paris, Albin Michel.

ISRAËL Stéphane \& MOCHON Jean-Philippe (1994). « Les Normaliens et la politique ». In SIRINELLI Jean-François (dir.). École normale supérieure. Le livre du bicentenaire. Paris, Presses universitaires de France : 191-213.

JoLy Marc (2018). « Naissance de l'“intellectuel collectif”? ». Biens symboliques/Symbolic Goods, 3. [Consulté le 01 avril 2021].

LABORIE Pierre (2011). Le Chagrin et le venin. La France sous l'Occupation, mémoire et idées reçues. Paris, Bayard. 
LACHAISE Bernard (2009). «L'entourage de Charles de Gaulle président du GPRF à Paris (25 août 1944-21 janvier 1946)». Histoire@politique. Politique, culture, société, 8. [Consulté le 01 avril 2021].

LECONTE Cécile (2019). « La Carrière militante du "grand remplacement" au sein du milieu partisan de l'Alternative pour l'Allemagne (AFD)». Politix, 126 : 111-134.

LE Quan-Dung (1948). « Cette puissance supérieure à toutes : l'esprit ». La Gauche, 10.

LEE Christopher J. (dir.) (2010). Making a World after Empire.The Bandung Moment and Its Political Afterlives. Athens, Ohio University Press.

MARIOT Nicolas (2017) [2013]. Tous unis dans la tranchée ? 1914-1918, les intellectuels rencontrent le peuple. Paris, Seuil.

MATONTI Frédérique (1996). « Les "Bricoleurs". Les cadres politiques de la raison historienne :

l'exemple de La Nouvelle critique ». Politix, 36 : 95-114.

MATONTI Frédérique \& POUPEAU Franck (2004). « Le Capital militant. Essai de définition ». Actes de la recherche en sciences sociales, $155: 4-11$.

MATONTI Frédérique (2005a). Intellectuels communistes : essai sur l'obéissance politique. « La Nouvelle Critique ", 1967-1980. Paris, La Découverte.

MATONTI Frédérique (2005b). « Arts, culture et intellectuels de gauche au XX ${ }^{\mathrm{e}}$ siècle ». In BECKER Jean-Jacques \& CANDAR Gilles (dir.). Histoire des gauches en France. $X X^{e}$ siècle : à l'épreuve de l'histoire, vol. 2. Paris, La Découverte : 684-702.

MATONTI Frédérique (2012). « Plaidoyer pour une histoire sociale des idées politiques ». Revue d'histoire moderne \& contemporaine, 59(4bis) : 85-104.

MILZA Pierre (1987). «Les Mouvements pacifistes et les guerres froides depuis 1947 ». Les Internationales et le problème de la guerre au XXe siècle. Actes du colloque de Rome (22-24 novembre 1984). Rome, École française de Rome : 265-283.

NOIRIEL Gérard (2018). Une Histoire populaire de la France. De la guerre de Cent Ans à nos jours. Marseille, Agone.

OFFERLÉ Michel (1987). Les Partis politiques. Paris, Presses universitaires de France, coll. « Que saisje ».

OFFERLÉ Michel (dir.) (1999). La Profession politique. XIX $-X X^{e}$ siècles. Paris, Belin.

PERROT Michelle (1993). Mémoires de 68. Guide des sources d'une histoire à faire. Paris, Verdier.

PEPY Daniel (1955). « Note sur le Mouvement républicain populaire ». In DUVERGER Maurice (dir.). Partis politiques et classes sociales en France, Cahiers de la Fondation nationale des sciences politiques, Série Partis et Élections, 74. Paris, Armand Colin : 209-218.

PUDAL Bernard (1989). Prendre parti. Pour une sociologie historique du PCF. Paris, Presses de la fondation nationale de sciences politiques.

PUDAL Bernard (2004). « Pour une histoire de l'“intellectuel collectif" communiste avec et contre les enseignants (1920-2000) ». In GIRAULT Jacques (dir.). Les Enseignants dans la société française au $X X^{e}$ siècle. Itinéraires, enjeux, engagements. Paris, Publications de la Sorbonne : 181-212.

PUDAL Bernard (2006). « De l'Histoire des idées à l'histoire sociale des idées politiques ». In COHEN Antonin, LACROIX Bernard \& RIUTORT Philippe (dir.). Les Formes de l'activité politique. Éléments d'analyse sociologique XVIII ${ }^{e}-X X^{e}$ siècle. Paris, Presses universitaires de France : 185-192. 
PUDAL Bernard (2007). « Le PCF, Gramsci et "l'intellectuel collectif" ». Intervention au colloque, « Antonio Gramsci, l'intellectuel aujourd'hui : politique, culture, globalisation », 25-27 octobre. Dudelange, Luxembourg.

PUDAL Bernard \& PENNETIER Claude (2014). « Introduction. Le "sujet" Communiste ». In PUDAL Bernard \& PENNETIER Claude (dir.). Le Sujet communiste. Identités militantes et laboratoires du « moi ». Rennes, Presses universitaires de Rennes.

RACINE Nicole \& TREBITSCH Michel (dir.) (1994). « Intellectuels engagés d'une guerre à l'autre ». Cahiers de l'IHTP, 26. Paris, CNRS Éditions.

RASSEMBLEMENT DÉMOCRATIQUe RÉVOLUTIONNAIRE (RDR) (1948). « Projet et plan de travail pour un programme du Rassemblement démocratique révolutionnaire ». La Gauche, $3: 4$.

REYNAUD-PALIGOT Carole (2001). Parcours politique des surréalistes. 1919-1969. Paris, CNRS Éditions.

RIMBERT Pierre (1955). « Le Parti socialiste S.F.I.O.». In DUVERGER Maurice (dir.). Partis politiques et classes sociales en France. Cahiers de la Fondation nationale des sciences politiques, Série Partis et Élections, 74. Paris, Armand Colin.

RIOUFREYT Thibault (2019). « La Mise en politique des idées. Pour une histoire sociale des idées en milieu partisan ». Politix, $126:$ 7-35.

RONSAC Charles (1948). « La Quinzaine dans le monde. Palestine, Afrique du Sud, Tchécoslovaquie... Mais où sont donc la Liberté et la Démocratie?». La Gauche, 2.

ROSENTHAL Gérard (1948). « D'une guerre absurde à la civilisation. Une Palestine libre doit être tête de pont du progrès socialiste dans le Proche-Orient ». La Gauche, 3.

ROUSSET David (1948). « Du déclin socialiste à l'isolement communiste. Un grand vide qu'il faut combler ». La Gauche, $2: 1$.

SAPIRO Gisèle (1999). La Guerre des écrivains, 1940-1953. Paris, Fayard.

SAPIRO Gisèle (2009). « Modèles d'intervention politique des intellectuels. Le cas français ». Actes de la recherche en sciences sociales, 176-177 : 8-31.

SAPIRO Gisèle (2018). Les Écrivains et la politique en France. De l'Affaire Dreyfus à la guerre d'Algérie. Paris, Seuil.

SARTRE Jean-Paul, ROUSSET David, ROSENTHAL Gérard (1949). Entretiens sur la politique. Paris, Gallimard.

SARTRE Jean-Paul [1949] (2012). « Présentation des Temps Modernes ». Les Temps Modernes, 1, octobre 1945. Repris dans SARTRE Jean-Paul. Situations II. Paris, Gallimard : 205-226.

SAWICKI Frédéric (1997). Les Réseaux du Parti socialiste. Sociologie d’un milieu partisan. Paris, Belin. SKINNER Quentin [1978] (2001). Les Fondements de la pensée politique moderne. Traduit de l'anglais par Jérôme Grossman et Jean-Yves Pouilloux. Paris, Albin Michel.

SKORNICKI Arnault \& TOURNADRE Jérôme (2015). La Nouvelle histoire des idées politiques. Paris, La Découverte.

SKORNICKI Arnaud \& GABORIAUX Chloé (dir.) (2017). Vers une histoire sociale des idées politiques. Villeneuve-d'Ascq, Presses universitaires du Septentrion.

SUBILEAU Françoise (1981). « Le Militantisme dans les partis politiques sous la Cinquième République : État des travaux de langue française ». Revue française de science politique, 31(5) : 1038-1068. 
TODD Olivier (2001). André Malraux. Une vie. Paris, Gallimard.

WRIGHT Richard (1947a). Un Enfant du pays. Traduit de l'anglais par Hélène Bokanowski et Marcel Duhamel. Paris, Albin Michel.

WRIGHT Richard [1940] (1947b). Black Boy. Traduit de l'anglais par Marcel Duhamel et Andrée R.

Picard. Paris, Gallimard.

\section{NOTES}

1. Disponibles à l'Office universitaire de recherches socialiste (Ours).

2. La Contemporaine, Bibliothèque, Archives, Musée des mondes contemporains à Nanterre. Section périodique, La Gauche, n 1-13, cote GF P 3660.

3. Archives départementales des Deux-Sèvres, Niort, 1426W4.

4. Principalement Paris, Lyon, Nancy, Toulouse et Bordeaux. À titre de comparaison, la part des habitant·e's urbain'e's en France était alors de 38,9 \% (Casevitz 1947 : 107).

5. Les professions déclarées par les militant·e's ont été agrégées selon la nomenclature de $1954-$ la plus proche de la période étudiée - à partir de leur intitulé dans la liste de membre du RDR exploitée. Les ratios sont calculés relativement aux résultats produits par Paul Clerc sur la période 1954-1962 (Clerc 1964).

6. La profession n'est pas renseignée pour trois des membres du CD et le dernier, Hubert Jean, est cultivateur dans la Manche.

7. Née le 19 octobre 1915 à Saint-Pierre-d'Albigny (Savoie) et morte à Ivry-sur-Seine le 14 avril 2011. Professeure agrégée de Lettres au lycée de Chartres, elle est également journaliste à Résistance, La Jeune République, en 1946 puis à L'agence européenne de presse et à Ce Matin, en 1947. Par la suite, elle enseigne au Lycée Fénelon à Paris. Candidate aux élections législatives du 10 novembre 1946 pour le Rassemblement de la gauche républicaine, elle est également membre de la Jeune République. Archives de la préfecture de police de Paris, Renseignements généraux, fiche individuelle, sur dérogation partielle, 77W 4179439.647.

8. Bertrand Geay, quarante ans plus tard, observe le même phénomène à propos des instituteurs et institutrices qu'il étudie (Geay 1999 : 21).

9. Selon l'expression de David Rousset (Rousset $1948: 1$ ).

10. Archives de la Préfecture de police de Paris, novembre 1948, Rassemblement démocratique révolutionnaire, dossier GaR2. À titre de comparaison, Franc-Tireur est diffusé à 370000 exemplaires environ, tandis que Le Figaro revendique la vente de 343664 exemplaires (Le Figaro, 20 mars 1948).

11. Cependant, sur les cinq textes fournis par Jean-Paul Sartre, seuls deux sont inédits, les autres sont des reprises d'autres publications.

12. Structure regroupant la France métropolitaine, les pays colonisés et les protectorats.

13. La scission entre la Confédération générale du Travail (CGT) et la Confédération générale du Travail-Force ouvrière (CGT-FO) date de décembre 1947. La position du RDR en faveur de l'unité syndicale et du rapprochement des tendances peut ainsi être rapprochée de celle de la Fédération de l'Éducation nationale (FEN), autonome depuis un vote interne en mars 1948.

14. Les citations suivantes sont tirées de «Appel du comité pour le Rassemblement démocratique révolutionnaire», Ronétypé, non daté (probablement février-mars 1948), Fonds Jean-René Chauvin, Centre d'histoire sociale du XX $\mathrm{X}^{\mathrm{e}}$ siècle, 1-JRC-3H.

15. Archives départementales des Deux-Sèvres, «Profession de foi de Charles Marché », mars 1949, dossier 1426W4.

16. Faute d'informations sur le tirage, il est difficile de mesurer l'éventuel succès du livre. 
17. Archives de la Préfecture de police de Paris, Renseignements généraux, "Confrontation publique des représentants de la culture mondiale organisée par le Rassemblement, Démocratique Révolutionnaire ", 14 décembre 1948, Rassemblement démocratique révolutionnaire, dossier GaR2.

18. "Pour la liberté et la fraternité de tous les peuples", Franc-Tireur, 13 décembre 1948, p. 2, souligné dans l'article.

19. L'alliance du RDR et de ses membres trotskistes avec l'Étoile nord-africaine de Messali Hadj est également explicable par la rupture de ce dernier d'avec le PCF en 1928 (Noiriel 2018 : 545).

\section{RÉSUMÉS}

Créé en France en 1948, le Rassemblement démocratique révolutionnaire (RDR) est une entreprise politique qui défend une position neutraliste de refus de l'alternative entre les blocs atlantiste et soviétique. Partant de la présentation sociographique des intellectuel-le·s engagée's dans la fondation de cette entreprise politique originale, cet article entend interroger la mobilisation et les prises de position politique de ce milieu partisan particulier, marqué par la surreprésentation des intellectuel-le·s. Il s'agira d'analyser les logiques de construction d'une position nouvelle au sein des champs politique et intellectuel, mais également la manière dont les formes d'intervention et les prises de position du RDR sont influencées par ses logiques sociales de recrutement. Finalement on se demandera comment les membres du Rassemblement interviennent à double titre au sein du champ de production idéologique : comme entreprise partisane et comme intellectuel collectif, et combien la recherche d'un si fragile équilibre peut peser dans la réussite ou non de leur entreprise.

The Rassemblement Démocratique Révolutionnaire [Revolutionary Democratic Rally] (RDR) was a political venture created in France in 1948 with the aim of defending a neutralist position, refusing the either-or alternatives between the Atlantic and Soviet blocs. This article begins with a sociographic presentation of the militant intellectuals who founded this original political initiative; then, we address the political activism and positionings of this distinctive partisan milieu, where intellectuals were characteristically overrepresented. We analyse the RDR's methods of establishing a new position within the political and intellectual fields, as well as the way in which their forms of intervention and positionings were influenced by their members' social profiles and trajectories as activists. Lastly, we question how the RDR's members intervened within the domain of ideological production - both as a partisan venture and a collective intellectual - and to what extent the search for such a fragile equilibrium impacted its success or failure.

\section{AUTEUR}

\section{BASTIEN AMIEL}

Université Paris Nanterre/Institut des sciences sociales du politique (ISP) 